\title{
Le rejet des eaux industrielles dans le bassin de la Molasse dans le sud de l'Allemagne : expérience de 27 années d'injection et conséquences
}

\author{
par K.-P. Seiler
}

de l'Institut GSF d'hydrologie

Oberschleißheim, Allemagne

\section{INTRODUCTION}

Au nord de Munich se retrouvent de grandes ressources de bentonite dans des couches miocènes. Elles sont exploitées et traitées pour leur restituer leur haute capacité d'absorption ; il en sort un produit qu'on utilise entre autres pour la production d'aliments, de peintures et de papiers spéciaux. Pour la réactivation de l'absorption de la bentonite on utilise des acides de chlorures qui libèrent la bentonite surtout des métaux lourds fixés à sa surface active. Dans les liquides résiduels se retrouvent les métaux lourds, les chlorures et une grande quantité de cations. Avant 1968 on rejetait les eaux résiduaires après une neutralisation et une sédimentation des matières en suspension, dans la rivière Isar et on diminuait ainsi fortement la qualité des eaux de surface. A son initiative, le producteur a été autorisé à injecter les eaux utilisées après filtration à $1000 \mathrm{~m}$ de profondeur dans le Malm sous la Molasse.

De 1968 jusqu'à aujourd'hui à peu près 42 millions de $\mathrm{m}^{3}$ d'eaux résiduaires ont été rejetées dans le Malm sans que le système d'injection ou l'aquifère n'ait jamais mal fonctionné. La base scientifique chimique fut crée avant le premier forage, mais la base hydrodynamique et le contrôle des données furent établis au cours des années quatre-vingt à quatre-vingt-dix. Le jugement du rejet était basé au début sur des considérations plutôt théoriques, qu'on a contrôlées au cours des 27 années systématiquement par l'installation de forages d'observation et par des observations régulières chimiques, géophysiques et hydrodynamiques et par l'évaluation numérique des données recueillies. Donc on dispose aujourd'hui d'une information suffisante pour juger des conséquences de ce rejet des eaux industrielles à court et long terme.

\section{LES DONNÉES GÉOLOGIQUES}

Entre le bord tectonique des Alpes et le Danube s'étend le bassin de la Molasse du sud de l'Allemagne (fig. 1). Etabli au Paléogène ce bassin accumulait continuellement les sédiments des Alpes émergentes jusqu’au Miocène. Cette sédimentation se produisait alternativement en milieu marin et fluvio-lacustre et produisait une prédominance de sédiments fins.

Dans la plus grande partie du bassin, le Malm (jurassique supérieur) forme la base des sédiments tertiaires; seulement à l'est se retrouvent entre le Malm et les sédiments tertiaires des grès et des argiles du Crétacé supérieur [1].

L'exploration des ressources en pétrole et gaz donnait la première base d'information géologique et hydrodynamique sur les ressources liquides dans le Tertiaire et le Malm. Elle créait aussi la base pour l'exploitation des eaux souterraines pour leur utilisation thérapeutique et pour la mise en œuvre d'installations souterraines pour le stockage de gaz. Un aspect spécial de la gestion provient de l'injection de liquides industriels à Moosburg. Cependant l'utilisation de la chaleur géothermique n'est pas encore bien avancée.

Le Malm sous la Molasse atteint une épaisseur entre 300 et $600 \mathrm{~m}$ et présente les mêmes faciès que le Malm franconien qui affleure immédiatement au nord du Danube (fig. l). Il s'est karstifié avant d'être couvert par des couches du Crétacé et du Tertiaire. Donc il existe un haut potentiel de perméabilité de la partie supérieur du Malm $\left(10^{-5} \mathrm{~m} / \mathrm{s}\right)$ et il en ressort qu'à peu près $60 \%$ des forages atteignant le Malm perdent leurs boues de forage et $40 \%$ n'ont même pas retrouvé des eaux.

Du nord au sud les sédiments tertiaires du bassin de la Molasse non chevauchés atteignent une épaisseur résiduelle de $1000 \mathrm{~m}$ jusqu'à $6000 \mathrm{~m}$ de Moosburg. Les sédiments tertiaires sont d'avantage argileux; seulement au Rupel, Chatt et Helvet on retrouve aussi des sables épais et des graviers sableux avec une perméabilité entre $10^{-4}$ et $10^{-6} \mathrm{~m} / \mathrm{s}$. Cependant $70 \%$ des sédiments fins du Tertiaire ont des perméabilités voisins de $10^{-10} \mathrm{~m} / \mathrm{s}$.

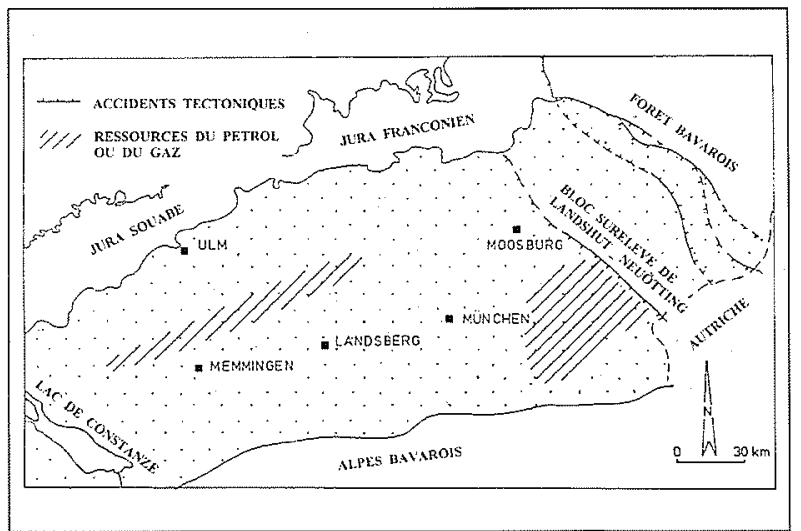

1. Le bassin de la Molasse au sud de l'Allemagne avec le lieu de rejet des eaux industrielles. 
Du point de vue tectonique (fig. I) on ne retrouve que des failles à petit rejet dans le bassin de la Molasse qui ne sont cependant plus actives. Donc en temps historique cette région ne connaît que très peu de tremblements de terre locaux qui ne dépassent pas cinq sur l'échelle de Mercalli.

\section{LES DONNÉES HYDROGÉOLO- GIQUES}

Les sédiments tertiaires superficiels représentent une ressource d'eaux potable très importante La recherche sur cette ressource a montré (fig. 2) que la zone active de la recharge, c'est-à-dire les eaux souterraines superficielles [2], se retrouvent entre 50 et $60 \mathrm{~m}$ au-dessous du niveau piézométrique. Dès cette profondeur les âges des eaux souterraines augmentent plus ou moins spontanément jusqu'à mille ans, la chimie de ces eaux montre un échange ionique et les eaux souterraines sont ici artésiennes; c'est-à-dire qu'on se retrouve dans la zone des eaux souterraines profondes (fig. 2). Les mesures qu'on a effectuées au cours de l'exploration pétrolière montrent en plus qu'on rencontre dans les environs de Moosburg entre 400 et $500 \mathrm{~m}$ de profondeur des eaux fossiles d'origine marine (fig. 2).

A Moosburg on retrouve les eaux souterraines dans le Malm dès une profondeur de $1000 \mathrm{~m}$. Le niveau piézométrique des eaux de l'aquifère du Malm, cependant, est plus bas que les niveaux correspondants des eaux souterraines superficielles ou profondes dans le Tertiaire (fig. 3). Cette piézométrie basse du Malm est connue dans toute la région au sud du Danube; elle est due à la haute perméabilité $\left(10^{-5} \mathrm{~m} / \mathrm{s}\right)$ du Malm comparée à celle des couches tertiaires et est liée à un écoulement souterrain vers le Danube (voir plus bas). L'aquifère du Malm fonctionne comme couche drainante pour les eaux dans les sédiments tertiaires et répond hydrodynamiquement de façon plus sensible aux changements du niveau d'écoulement du Danube que les eaux dans les aquifères tertiaires. Donc le système hydrodynamique du Malm possède aujourd'hui sa sous-pression (fig. 3) à cause de la forte érosion du Danube dans le passé géologique.

Dans la partie centrale du bassin de la Molasse le système hydrodynamique du Malm se retrouve dans un état transitoire, c'est-à-dire qu'il se vide plus vite qu'il n'est rechargé par les eaux souterraines des aquifères tertiaires. Plusieurs profils géothermiques ont été enregistrés dans les forages de Moosburg, qui montrent clairement cet écoulement transitoire.

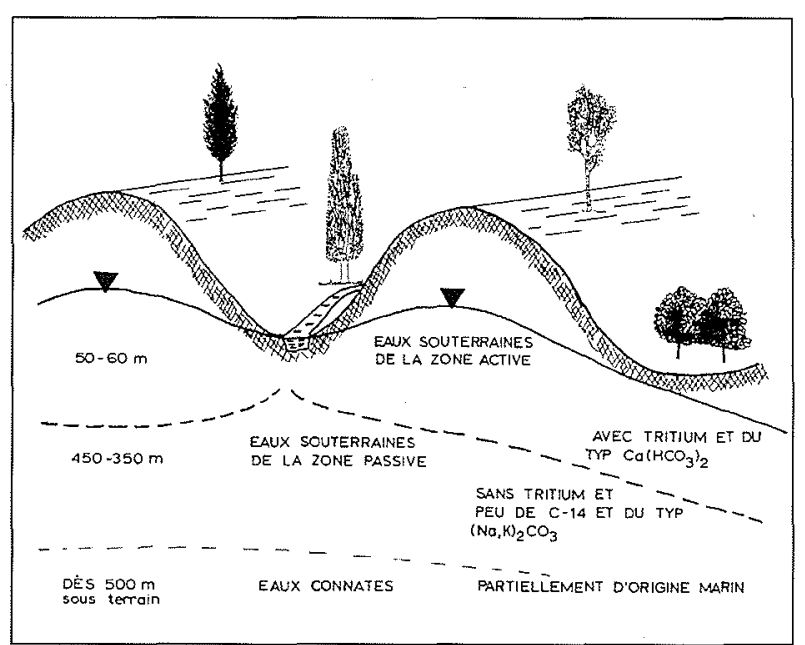

2. La subdivision des eaux souterraines de la région de Moosburg selon la participation dans le cycle d'eau.

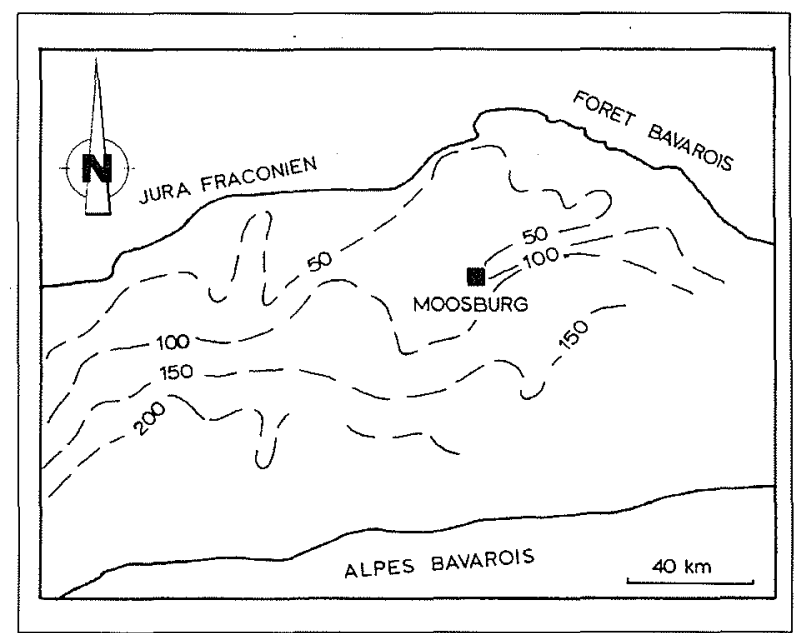

3. La sous-pression des eaux souterraines dans le Malm en mètres de colonne d'eau par rapport au niveau des cours d'eau (après [3])

Dans le cas du champ géothermique non perturbé (fig. 4 , SC IV) les températures augmentent continuellement avec la profondeur, au niveau du toit du Malm plus vite qu'au-dessus, et ne changent que très peu dans le Malm. Evidemment les températures dans le Malm sont apportées de manière convective des régions plus profondes et se traduisent de manière conductive aux couches de base du Tertiaire ; une infiltration d'eau du Malm est exclue du fait que le Malm représente un régime hydrodynamique de sous-pression.

Dans le cas où les températures dans le Malm ont diminué par l'injection des eaux industrielles de $33{ }^{\circ} \mathrm{C}$ (fig. 4, SC III) et où aucune injection n'a pas encore eu lieu dans le forage, on observe dans les couches tertiaires la même aug-

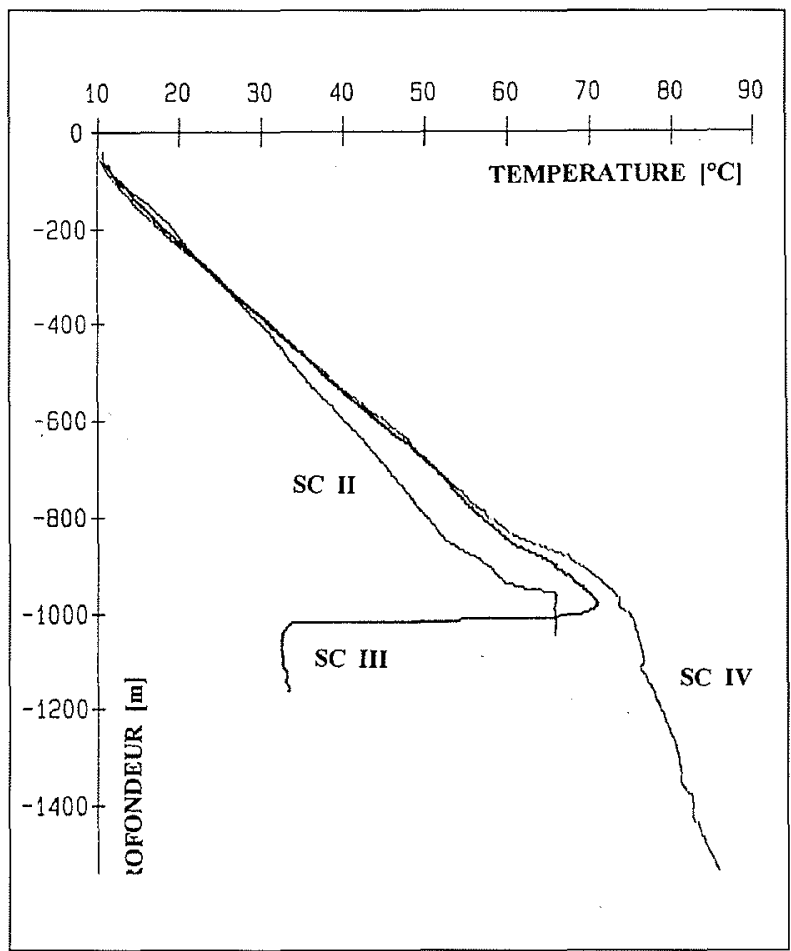

4. Profils géothermiques de trois forages à Moosburg. Profil SC III de 1983, profils SC II et IV de 1991. 
mentation des températures que dans le SC IV, mais un recul des températures dans la région basse du Tertiaire qui est aussi due à un échange plutôt conductif que convectif.

Comparant les profils géothermiques des forages SC III et IV avec celui du forage SC II on remarque des températures qui augmentent dans le Tertiaire moins vite avec la profondeur (fig. 4, SC II). En effet on a injecté dans le forage SC II pendant dix ans continuellement des eaux froides; ensuite ce forage fut transformé en forage d'observation et l'enregistrement du profil géothermique a eu lieu dix ans après la transformation. Evidemment les apports convectifs n'ont pas changé entre-temps fortement les températures.

En conséquence des observations géophysiques les apports d'eaux souterraines du Tertiaire au Malm doivent être très petits comparés au flux dans le Malm lui-même. Cependant une certaine influence des eaux soutemaines du Tertiaire sur les écoulement du Malm est décelée par le fluor, le soufre et le méthane qui datent plutôt du Tertiaire que du Malm. Cette interprétation implique aussi que la diminution de la pression hydraulique dans le Malm a causé probablement pendant une grande partie du tertiaire la destruction de ressources en hydrocarbones et serait alors une raison possible pour les faibles ressources en pétrole et gaz dans la partie préalpine du bassin de la Molasse allemande (fig. 1).

La piézométrie pour les eaux de l'aquifère du Malm (fig. 5) montre un écoulement des eaux souterraines de Moosburg en direction NE vers le Danube dans la région de Regenburg-Straubing [3]. Cette zone de résurgence se retrouve à $60 \mathrm{~km}$ de Moosburg. Avec les données hydrodynamiques de l'aquifère (perméabilité de $10^{-5} \mathrm{~m} / \mathrm{s}$, gradient hydraulique de $0,1 \%$ et porosité efficace de $2 \%$ ) on calcule un temps de résidence des eaux de Moosburg jusqu'à la zone de résurgence de quelques mille à dix mille ans; dans ce temps les eaux injectées accroîtront dans le Danube la concentration en chlorures (voir chapitre 6) en temps d'étiage au maximum de $6 \mathrm{mg} / \mathrm{l}$ et en temps d'écoulement moyen de $3 \mathrm{mg} / \mathrm{l}$.

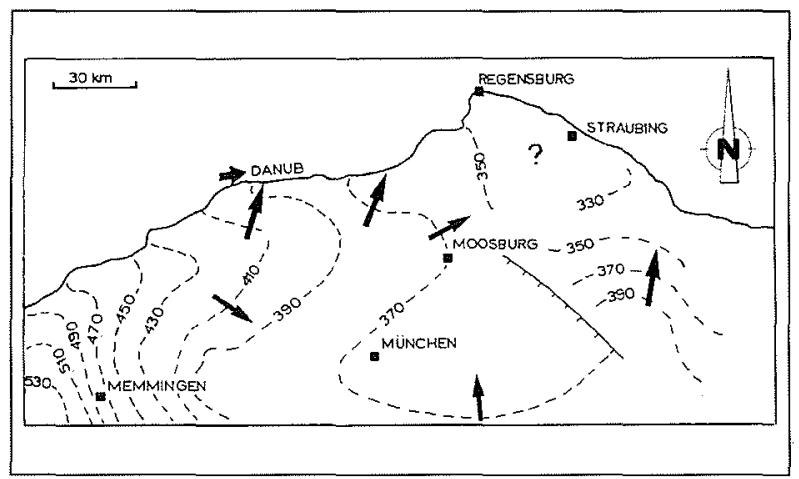

5. Carte piézométrique des eaux souterraines dans le Malm sous la Molasse (après [3]). Lignes équipotentielles en m s.n.m; flèches : direction d'écoulement souterrain.

\section{LES CONDITIONS PRÉALABLES POUR L'INJECTION DE LIQUIDES INDUSTRIELLES DANS LES COUCHES PROFONDES}

Les conditions préalables pour l'injection d'eaux industrielles dans les couches profondes ont été définies par [4], [5], [6]. A l'égard de la situation à Moosburg ces conditions sont garanties et on dispose même d'une sécurité addition- nelle; c'est ainsi qu'il est absolument exclu que des captages d'eaux potables puissent être affectés par le rejet.

- La couche d'injection se trouve couverte de plus de $500 \mathrm{~m}$ de couches argileuses d'une perméabilité de $10^{-10} \mathrm{~m} / \mathrm{s}$ remplies d'eaux fossiles.

- Les caractéristiques hydrodynamiques de la couche d'injection suffisent pour recueillir et stocker les eaux injectées. Jusqu'à aujourd'hui le rejet de $42106 \mathrm{~m}^{3}$ a produit une augmentation locale du niveau piézométrique $(<1 \mathrm{~km})$ de $38 \mathrm{~m}$ sans atteindre le niveau du terrain. Cette augmentation n'a pas changé dans les dernières 27 années.

- On ne se trouve pas dans une zone sismique.

- Dès $500 \mathrm{~m}$ de profondeur les aquifères ne peuvent plus servir comme ressource d'eau potable, même si la chimie des eaux le permettait, parce qu'il manque la recharge et les ressources en eaux exploitables dans cette région sont suffisamment grandes et peuvent être protégées.

- Le système hydrodynamique dans le Malm appartient à un écoulement transitoire et de sous-pression; donc il n'y a absolument pas d'échange avec les aquifères exploités pour l'eau potable à des distances proches ou loin de Moosburg.

En quelques endroits de l'est du bassin de la Molasse on utilise en petites quantités les eaux souterraines du Malm comme moyen thérapeutique; ces ressources ne seront jamais touchées par l'injection d'eaux industrielles à Moosburg, parce qu'elles se retrouvent hydrodynamiquement séparées. L'utilisation des ressources géothermiques liées aux Malm ne serait possible qu'en réinjectant des eaux froides pour garantir une gestion d'eaux en équilibre avec la recharge qui n'existe pas; elle ressemblerait en quelque sorte à l'injection des eaux industrielles à Moosburg.

\section{LES INSTALLATIONS D'INJECTION ET LES QUANTITÉS INJECTÉES}

A Moosburg existent quatre forages (fig. 6) qui entrent dans le Malm à environ $1000 \mathrm{~m}$ sous terre. Trois forages pénètrent le Malm entre 100 et $300 \mathrm{~m}$ (SC I, II, III) et le quatrième de $600 \mathrm{~m}$ jusqu'à sa base (SC IV). L'analyse des carottes mène à la conclusion que le Malm possède seulement sur les premiers $300 \mathrm{~m}$ sous son toit une perméabilité remarquable; en dessous il manque de porosité

Tous les forages sont crépinés dans le Malm et portent des tubes cimentés dans tout le Tertiaire. Dans l'espace annulaire extérieur des forages on a installé des sondes pour le contrôle de la conductivité et du niveau statique derrière les tubes.

Deux forages servent à l'injection (SC I et SC III, fig. 6) et deux à l'observation des niveaux statiques et de la composition chimique et isotopique des eaux du Malm (SC II et SC IV, fig. 6).

\section{LA CHIMIE DES EAUX SOUTER- RAINES ET LES RÉACTIONS DE NEUTRALISATION}

Dans le passé le forage III était d'abord un forage d'observation et le forage II un forage d'injection, aujourd'hui c'est l'inverse.

Le propriétaire de l'usine de Moosburg a l'autorisation d'injecter au maximum $1,7510^{6} \mathrm{~m}^{3} / \mathrm{an}$. Entre 3968 et 1995 $42106 \mathrm{~m}^{3}$ ont été injectés. Si on néglige toute dispersion hydrodynamique et la dissolution de carbonates en conséquence de la neutralisation des eaux acides (voir chapitre 6) les quantités injectées occupent dans l'aquifère du Malm un cylindre avec un rayon de $1493 \mathrm{~m}$ 


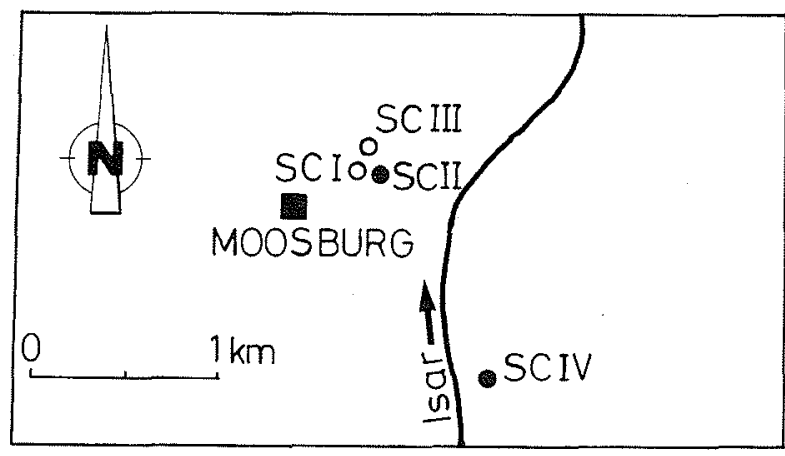

6. Les forages de Moosburg. Cercles noirs : forages d'observation, cercles ouverts : forages de rejet.

Il existe des analyses chimiques et isotopiques des eaux vierges du Malm en différents endroits de la zone centrale du bassin de la Molasse. Les résultats des analyses sont tellement similaires qu'il suffit d'en reporter dans le tableau ? celles du forage SC IV. Du point de vue du soufre, du fluor, de la température et de l'oxygène, c'est-à-dire du point de vue sanitaire elles n'ont pas la qualité d'eaux potables.

La composition chimique des eaux injectées varie selon le cycle de fabrication. La composition moyenne est présentée dans le tableau 1 ; ces eaux sont surtout riches en cations, chlore, en métaux lourds, en fer et en aluminium. Leur poids spécifique est très peu élevé $(1,02 \mathrm{~kg} / \mathrm{l})$ par rapport à l'eau chaude dans le Malm de Moosburg $(0,9778 \mathrm{~kg} / \mathrm{l})$.

Les forages d'observation touchés par le rejet montrent que la neutralisation des eaux résiduaires se produit très vite et à courte distance dans l'aquifère (tabl. l). Les réactions chimiques principales sont :

1. la neutralisation des carbonates en deux étapes en régime $\mathrm{pH}$ moins de 6,5

$\mathrm{CaO}_{3}+2 \mathrm{H}_{3} \mathrm{O}^{+}=\mathrm{Ca}^{2}+\mathrm{CO}_{2} \mathrm{aq}+3 \mathrm{H}_{2} \mathrm{O}$ en régime $\mathrm{pH}$ au dessus de 6,5 et moins de 10

$2 \mathrm{CaCO}_{3}+2 \mathrm{H}_{3} \mathrm{O}+=\mathrm{Ca}\left(\mathrm{HCO}_{3}\right)_{2}+\mathrm{Ca}^{2+}+\mathrm{H}_{2} \mathrm{O}$

2. l'immobilisation d'aluminium

en régime $\mathrm{pH}$ entre 5,5 et 7 [7]

$$
\mathrm{Al}^{3+}+6 \mathrm{HOH}=\mathrm{Al}(\mathrm{OH})_{3}+3 \mathrm{H}_{3} \mathrm{O}^{+}
$$

Tableau 1 - La composition chimique des eaux vierges du Malm (SC IV) et des eaux injectées avant et après la neutralisation dans le Malm.

\begin{tabular}{|c|c|c|c|c|}
\hline \multirow[b]{2}{*}{ Elément } & \multirow[b]{2}{*}{ Dimension } & \multirow[b]{2}{*}{ SC IV } & \multicolumn{2}{|c|}{ Les eaux injectées } \\
\hline & & & \multicolumn{2}{|c|}{ Neutralisation } \\
\hline $\mathrm{pH}$ & - & 7,3 & 0,9 & 5,9 \\
\hline $\mathrm{Ca}^{2+}$ & $\mathrm{mg} / \mathrm{l}$ & 40,7 & 1300 & 4930 \\
\hline $\mathrm{Mg}^{2+}$ & $\mathrm{mg} / \mathrm{l}$ & 13,0 & 1520 & 2080 \\
\hline $\mathrm{K}^{+}$ & $\mathrm{mg} / \mathrm{l}$ & 12,1 & 76 & 82 \\
\hline $\mathrm{Na}^{+}$ & $\mathrm{mg} / \mathrm{l}$ & 138 & 82 & 76 \\
\hline $\mathrm{F}^{-}$ & $\mathrm{mg} / \mathrm{l}$ & 2,9 & p.d. & p.d. \\
\hline $\mathrm{Cl}^{-}$ & $\mathrm{mg} / \mathrm{l}$ & 97 & 34000 & 17100 \\
\hline $\mathrm{SO}_{4}{ }^{2-}$ & $\mathrm{mg} / \mathrm{l}$ & 0,4 & 22 & 13 \\
\hline $\mathrm{As}^{4}$ & $\mathrm{mg} / \mathrm{l}$ & $<0,4$ & p.d. & 0,05 \\
\hline $\mathrm{Cd}$ & $\mathrm{mg} / \mathrm{l}$ & 4 & p.d. & 0,001 \\
\hline $\mathrm{Cr}$ & $\mathrm{mg} / \mathrm{l}$ & 29 & 2,8 & 0,004 \\
\hline $\mathrm{Cu}$ & $\mathrm{mg} / \mathrm{l}$ & 11 & 1,6 & 0,001 \\
\hline $\mathrm{Ni}$ & $\mathrm{mg} / \mathrm{l}$ & $<5$ & 2,7 & 0,005 \\
\hline $\mathrm{Pb}$ & $\mathrm{mg} / \mathrm{l}$ & 49 & 0,7 & 0,002 \\
\hline $\mathrm{Se}$ & $\mathrm{mg} / \mathrm{l}$ & $<0,4$ & p.d. & p.d. \\
\hline $\mathrm{Zn}$ & $\mathrm{mg} / \mathrm{l}$ & 24 & 6 & 0,08 \\
\hline Al & $\mathrm{mg} / \mathrm{l}$ & $<0,1$ & 4100 & $<0,1$ \\
\hline $\mathrm{Fe}$ & $\mathrm{mg} / \mathrm{l}$ & p.d. & 2770 & 0,61 \\
\hline
\end{tabular}

3. l'immobilisation du fer

$$
\mathrm{Fe}^{2+}+2 \mathrm{HOH}=\mathrm{Fe}(\mathrm{OH})^{+}+\mathrm{H}_{3} \mathrm{O}^{+}
$$

L'immobilisation de l'aluminium et du fer libère des cations qui eux aussi entrent en réaction du type de l'équation 1 et 2. Les précipités des hydroxydes du fer et surtout de l'aluminium ont une très haute capacité d'absorption et donc coprécipitent tous les métaux lourds. Il en résulte que les eaux injectées se transforment vite en eaux riches en calcium, magnésium, bicarbonate, $\mathrm{CO}_{2}$ et chlorures ; ces éléments influencent la qualité d'eaux du Malm. Cependant les éléments toxiques ne jouent plus un rôle, parce qu'ils précipitent sur place et y restent immobiles pour toujours.

L'augmentation de la porosité de l'aquifère par les processus de neutralisation diminue les vitesses des flux et augmentent le stockage sur place. Donc les conditions limites hydrodynamiques dépendent fortement des conditions chimiques.

La solution des carbonates est calculée sur la base des équations 1 à 4 et

$17 \mathrm{~g} \mathrm{CaCO}_{3}$ par $0,17 \mathrm{~mol} / \mathrm{l}$ de cations (voir équation 2)

$45 \mathrm{~g} \mathrm{CaCO}_{3}$ par $0,45 \mathrm{~mol} / \mathrm{l}$ de cations libérés par la réaction de l'aluminium (voir équation 3 et 2 ) et

$5 \mathrm{~g} \mathrm{CaCO}_{3}$ par $0,05 \mathrm{~mol} / \mathrm{l}$ de cations libérés par la réaction du fer (voir équation 4 et 2 ).

Ces valeurs de solution sont seulement approximatives, parce qu'on ignore si le $\mathrm{CO}_{2}$ reste piégé dans l'eau et quelle est l'appréciation exacte du fer.

Quelle que soit la valeur correcte des carbonates dissous, on doit supposer qu'il existe autour des lieux d'injection d'abord une zone de très haute porosité (fig. 6 , SC I et III) qui évolue continuellement dans la zone des porosités normales (fig. 6, de SC II à SC IV). Dans cet espace transitoire des porosités le mécanisme de la neutralisation s'intensifie avec la décroissance de la porosité.

Avec les valeurs de solution des carbonates de aquifères on calcule au maximum une solution de $2,810^{6} t$ de carbonates par $4210^{6} \mathrm{~m}^{3}$ d'eaux injectées. Il en résulte une caverne théorique de $300 \mathrm{~m}$ de longueur et d'un diamètre de $66 \mathrm{~m}$, qui est incapable de produire des tremblements de terre en s'effondrant parce que elle est trop petite et couverte de $1000 \mathrm{~m}$ de sédiments rigides.

\section{L LA PROPAGATION DES EAUX NEU- TRALISÉES SOUS MOOSBURG}

Les essais de pompage dans le Malm à Moosburg et en d'autres endroits dans le bassin de la Molasse mènent toujours à une perméabilité de $10^{-5} \mathrm{~m} / \mathrm{s}$. La porosité efficace a été estimée à $2 \%$ selon les recherches dans le Malm franconien; son augmentation par les processus de neutralisation n'a pas été prise en considération. Par les analyses géologiques comme hydrauliques l'épaisseur hydrodynamiquement efficace de l'aquifère a été déterminée à $300 \mathrm{~m}$. Les dispersivités pour le Malm sont connues pour le Malm franconien [8] et s'appliquent aussi pour le Malm sous la Molasse parce que les deux régions avaient les mêmes conditions de sédimentation et après l'émergence du Malm la même karstification.

La combinaison de l'équation du flux et du transport pour calculer le transport des rejets montre que le corps d'eau injectée devrait avoir aujourd'hui une extension de $800 \mathrm{~m}$ comme rayon autour du centre d'injection (fig. 7). En effet le forage SC IV (fig. 3) qui est situé au sud-est de Moosburg à une distance de $1000 \mathrm{~m}$ n'a pas encore reçu les eaux neutralisées. Cependant il est bien probable que le front du cylindre de propagation arrivera dans un futur proche à ce point d'observation. Cette confirmation est souhaitée pour encore mieux juger si la base des calculs pour le karst sous la Molasse doit être rectifiée. 


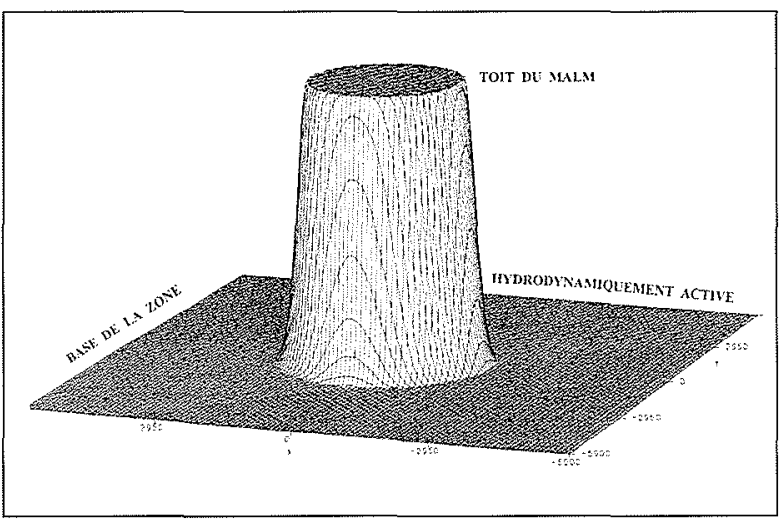

7. La géométrie du corps de propagation dans le Malm en-dessous de Moosburg après 27 ans de rejet. La hauteur du corps correspond à l'épaisseur hydrodynamiquement efficace. Le centre du corps est à peu près éloigné de $20 \mathrm{~m}$ du centre de rejet. Quantité d'injection $60 \mathrm{l} / \mathrm{s}$, conductivité hydraulique $10^{-5} \mathrm{~m} / \mathrm{s}$, porosité 0,02 .

\section{VIII 糃 CONCLUSIONS}

Le rejet des eaux industrielles est effectué dans le Malm sous la Molasse qui appartient à un système hydrodynamiquement ouvert et en état d'écoulement transitoire. L'aquifère de rejet dispose d'une perméabilité et d'une capacité de stockage suffisamment élevées; c’est ainsi qu'aucun problème d'injection ne s'est posé pendant les 27 années d'opération.

Le système hydrodynamiquement ouvert se trouve bien isolé des systèmes aquifères exploités pour les eaux potables. Donc il n'y a aucune influence négative à signaler jusqu'à maintenant et dans un futur historique.

Par faute de recharge appréciable dans le Malm sous la Molasse, ces eaux souterraines ne peuvent servir pour l'utili- sation de la chaleur géothermique. Cette utilisation devrait être connectée avec une réinjection d'eaux froides pour ne pas user la ressource d'eau. Cette réinjection mènerait aux mêmes phénomènes en profondeur que le rejet des eaux industrielles avec la différence que les eaux vont réacquérir en profondeur après un parcours plus ou moins long, la chaleur. C'est-à-dire que l'utilisation de l'aquifère du Malm comme source géothermique entrânerait un peu moins de restriction régionale que le rejet des eaux industrielles.

\section{BIBLIOGRAPHIE}

[1] Lemcke K. (1988), - Das Bayerische Alpenvorland vor der Eiszeit. Geologie von Bayern I.r 175 S. (Schweitzerbart) Stuttgart.

[2] Seller K.-P. \& Lindner W. (1995). - Near-surface and deep groundwaters. J. Hydrol., 165, 33-44, Amsterdam.

[3] ANdres G. \& Frisch H. (1981). - Hydrogeologie und Hydraulik im Malmkarst des Molassebeckens und der angrenzenden schwäbisch-fränkischen Alb. Schriftenreihe Bayer. Landesamt f. Wasserwirtsch., 15, 108-116, München.

[4] WARNER D.L. (1968). - Subsurface disposal of liquid insuatrial wastes by deep well injection. Am. Assoc. Petroleum Geologists Mem 10, 11-20, Tulsa.

[5] Aust H. \& Kreysing K. (1978). - Geologische und geotechnische Grundlagen zur Tiefenversenkung von flüssige n Abfällen und Abwässern. Geol. Jahrb. C 20, 224 p., Hannover.

[6] Lamoreaux P.E. \& VRBA J. (1990). - Hydrogeology and management of hazardous waste by deep well disposal, 136 p. (IAH), Hannover.

[7] Stumm W. \& Morgan J.J. (1981). - Aquatic chemistry (Wiley \& Sons), New York.

[8] Seiler K.-P., Maloscewski P. \& Behrens H. (1989). Hydrodynamic dispersion in karstified limestones and dolomites in the upper Jurassic of the Franconian Alb, F.R.G. J Hydrol., 108, 235-247, Amsterdam. 